\title{
ON A PROBLEM OF TURÁN ABOUT POLYNOMIALS WITH CURVED MAJORANTS
}

\author{
BY
}

Q. I. RAHMAN

\begin{abstract}
Let $\phi(x) \geqq 0$ for $-1 \leqq x \leqq 1$. For a fixed $x_{0}$ in $[-1,1]$ what can be said for $\max \left|p_{n}^{\prime}\left(x_{0}\right)\right|$ if $p_{n}(x)$ belongs to the class $P_{\phi}$ of all polynomials of degree $n$ satisfying the inequality $\left|p_{n}(x)\right| \leqq \phi(x)$ for $-1 \leqq x \leqq 1$ ? The case $\phi(x)=1$ was considered by A. A. Markov and S. N. Bernstein. We investigate the problem when $\phi(x)=\left(1-x^{2}\right)^{1 / 2}$. We also study the case $\phi(x)=|x|$ and the subclass consisting of polynomials typically real in $|z|<1$.
\end{abstract}

The following theorem was proved by A. A. Markov in 1889.

THEOREM A. If $p_{n}(x)$ is a polynomial of degree $n$, such that $\left|p_{n}(x)\right| \leqq 1$ for $-1 \leqq x$ $\leqq 1$, then

$$
\max _{-1 \leqq x \leqq 1}\left|p_{n}^{\prime}(x)\right| \leqq n^{2} .
$$

The original paper [9] of Markov is not readily accessible but an excellent account of this and other related results is presented in [4]. In Theorem A equality is attainable only at \pm 1 and only for $p_{n}(x)=e^{i \gamma} T_{n}(x)$ where

$$
\begin{aligned}
T_{n}(x) & =\cos \left(n \cos ^{-1} x\right)=2^{n-1} \prod_{1}^{n}\left\{x-\cos \left(\left(\nu-\frac{1}{2}\right) \pi / n\right)\right\} \\
& =\frac{n}{2} \sum_{m=0}^{[n / 2]}(-1)^{m} \frac{(n-m-1) !}{m !(n-2 m) !}(2 x)^{n-2 m}
\end{aligned}
$$

is the so-called Chebyshev polynomial of the first kind.

For points $x$ lying in the interval $|x|<\cos \left(\frac{1}{2} \pi / n\right)$ the following theorem of Bernstein [2] gives a better estimate for $\left|p_{n}^{\prime}(x)\right|$.

THEOREM B. Under the conditions of Theorem A

$$
\left|p_{n}^{\prime}(x)\right| \leqq n\left(1-x^{2}\right)^{-1 / 2}, \quad-1<x<1 .
$$

This dominant $n\left(1-x^{2}\right)^{-1 / 2}$ is the best possible dominant only at the points $x=\cos \{(2 k+1) \pi /(2 n)\}, k=1,2, \ldots, n-1$. It is, however, asymptotically equal to the precise bound at every fixed point in the interior of the interval as $n$ becomes infinite.

Received by the editors February 1, 1971.

AMS 1970 subject classifications. Primary 30A06, 30A40; Secondary 42A04.

Key words and phrases. Polynomials with curved majorants, Chebyshev polynomial of the first kind, Chebyshev polynomial of the second kind, polynomials typically real in $|z|<1$, GaussLucas theorem.

Copyright (C) 1972, American Mathematical Society 
At a conference on Constructive Function Theory held in Varna, Bulgaria, Professor P. Turán proposed the following problem:

Problem. For any $x_{0}$ in $[-1,1]$ determine $\max \left|p_{n}^{\prime}\left(x_{0}\right)\right|$ for all polynomials $p_{n}(x)$ of degree $\leqq n$ satisfying the restriction

$$
\max _{-1 \leqq x \leqq 1} \frac{\left|p_{n}(x)\right|}{\sqrt{ }\left(1-x^{2}\right)}=1
$$

He remarked that even the value of $\max _{-1 \leqq x \leqq 1}\left|p_{n}^{\prime}(x)\right|$ did not seem to be known for the class in (3).

For real-valued polynomials the hypothesis says that the graph of $p_{n}(x)$ on the interval $-1<x<1$ is contained in the closed unit disk.

Let $\pi_{n}$ denote the class of polynomials $p_{n}(x)$ of degree $n$ which satisfy $\left|p_{n}(x)\right|$ $\leqq\left(1-x^{2}\right)^{1 / 2}$ for $-1<x<1$.

In looking for the maximum of $\left|p_{n}^{(k)}\left(z^{*}\right)\right|$ (the $k$ th derivative at a given point) for all polynomials $p_{n}(x)$ of degree at most $n$ which satisfy $\left|p_{n}(x)\right| \leqq 1$ for $-1 \leqq x \leqq 1$ it is enough to consider the subclass $A_{n}$ whose members are in addition real on the real axis. Let $p_{n}^{(k)}\left(z^{*}\right)=e^{i \gamma}\left|p_{n}^{(k)}\left(z^{*}\right)\right|$ and let $e^{-i \gamma} p_{n}(z)=p_{n, 1}(z)+i p_{n, 2}(z)$ where $p_{n, 1}$ and $p_{n, 2}$ are elements of $A_{n}$. Since $p_{n, 1}^{(k)}\left(z^{*}\right)=\left|p_{n}^{(k)}\left(z^{*}\right)\right|$ the maximum of $\left|p_{n}^{(k)}\left(z^{*}\right)\right|$ is attained, if at all, for some $p_{n}$ in $A_{n}$. The following theorem of Duffin and Schaeffer [7, p. 240] shows that the functions in $A_{n}$ are uniformly bounded on every compact set. Hence [1, p. 216] the maximum of $\left|p_{n}^{(k)}\left(z^{*}\right)\right|$ is, in fact, attained.

THEOREM C. Let $p_{n}(z)$ be a polynomial of degree $n$ or less such that in the real interval $(-1,1)\left|p_{n}(z)\right| \leqq 1$. Then for $z$ lying on the ellipse $\mathscr{E}_{R}$ with foci at $-1,+1$ and semiaxes $\frac{1}{2}\left(R+R^{-1}\right), \frac{1}{2}\left(R-R^{-1}\right)$, we have $\left|p_{n}(z)\right| \leqq \frac{1}{2}\left(R^{n}+R^{-n}\right)$.

For precisely the same reason as above the maximum of $\left|p_{n}^{(k)}\left(z^{*}\right)\right|$ over the class $\pi_{n}$ is attained for a polynomial which is real on the real axis.

We prove

THEOREM 1. If $p_{n}(x)$ is a polynomial of degree $n$ such that $\left|p_{n}(x)\right| \leqq\left(1-x^{2}\right)^{1 / 2}$ for $-1<x<1$, then

If

$$
U_{n}(x)=\left(1-x^{2}\right)^{-1 / 2} \sin \left\{(n+1) \cos ^{-1} x\right\}=\sum_{m=0}^{[n / 2 j}(-1)^{m} \frac{(n-m) !}{m !(n-2 m) !}(2 x)^{n-2 m}
$$

is the nth Chebyshev polynomial of the second kind then $p_{n}(x)=\left(1-x^{2}\right) U_{n-2}(x)$ satisfies the conditions of Theorem 1 and $\left|p_{n}^{\prime}( \pm 1)\right|=2(n-1)$. Hence the result is best possible.

THEOREM 2. Under the conditions of Theorem 1

$$
\left|p_{n}^{\prime}(x)\right| \leqq\left\{x^{2}\left(1-x^{2}\right)^{-1}+(n-1)^{2}\right\}^{1 / 2}, \quad-1<x<1 .
$$


The example $p_{n}(x)=e^{i \gamma}\left(1-x^{2}\right) U_{n-2}(x)$ shows that in (5) equality can be attained at those points of the interval $-1<x<1$ where $(n-1)\left(1-x^{2}\right)^{1 / 2} \tan \left\{(n-1) \cos ^{-1} x\right\}$ $=x$.

It follows from Theorem B that if $p_{n}(x)$ is a polynomial of degree $n$ such that $\left|p_{n}(x)\right| \leqq 1$ for $-1 \leqq x \leqq 1$ then $n^{-1}\left(1-x^{2}\right) p_{n}^{\prime}(x) \in \pi_{n+1}$. Hence we have the following corollary of Theorem 2 .

COROLlaRY 1. If $p_{n}(x)$ is a polynomial of degree $n$ such that $\left|p_{n}(x)\right| \leqq 1$ for -1 $\leqq x \leqq 1$, then

$$
\left|\left(1-x^{2}\right) p_{n}^{\prime \prime}(x)-2 x p_{n}^{\prime}(x)\right| \leqq n\left\{x^{2}\left(1-x^{2}\right)^{-1}+n^{2}\right\}^{1 / 2}, \quad-1<x<1 .
$$

When $x=0$ inequality (5) may be restated as follows:

If $p_{n}(x)=\sum_{k=0}^{n} a_{k} x^{k} \in \pi_{n}$ then $\left|a_{1}\right|=\left|p_{n}^{\prime}(0)\right| \leqq n-1$. This inequality is sharp for odd

$n$. Here again the extremal polynomial is $e^{i \gamma}\left(1-x^{2}\right) U_{n-2}(x)$.

We also estimate $\left|a_{2}\right|$.

THEOREM 3. If $p_{n}(x)=\sum_{k=0}^{n} a_{k} x^{k} \in \pi_{n}$ then

$$
\left|a_{2}\right| \leqq\left\{(n-1)^{2}+1\right\} / 2 \text {. }
$$

For even $n$ the bound in (7) is attained when $p_{n}(x)=e^{i \gamma}\left(1-x^{2}\right) U_{n-2}(x)$.

The next theorem is a refinement of Theorem $\mathrm{C}$ for polynomials belonging to $\pi_{n}$.

THEOREM 4. If $p_{n}(z) \in \pi_{n}$ then for $z$ lying on the ellipse $\mathscr{E}_{R}$ with foci at the points $-1,+1$ and semiaxes $\frac{1}{2}\left(R+R^{-1}\right), \frac{1}{2}\left(R-R^{-1}\right)$, we have

$$
\left|p_{n}(z)\right| \leqq\left|1-z^{2}\right|^{1 / 2} \frac{1}{2}\left(R^{n-1}+R^{-n+1}\right) .
$$

The problem of Turán mentioned earlier is a special case of the following general question subsequently asked by him:

Let $\phi(x) \geqq 0$ for $-1 \leqq x \leqq 1$. For a fixed $x_{0}$ in $[-1,1]$ what can be said for $\max \left|p_{n}^{\prime}\left(x_{0}\right)\right|$ if $p_{n}(x)$ belongs to the class $P_{\phi}$ of all polynomials of degree $\leqq n$ satisfying the inequality $\left|p_{n}(x)\right| \leqq \phi(x)$ for $-1 \leqq x \leqq 1$.

We shall consider only the simple class $P_{|x|}$ of polynomials $p_{n}(x)$ of degree $\leqq n$ which are dominated by the function $|x|$ on $[-1,1]$.

If $p_{n}(z)$ is a polynomial of degree $n$ such that $\left|p_{n}(x)\right| \leqq|x|$ for $-1 \leqq x \leqq 1$ then $p_{n}(z)=z g_{n-1}(z)$ where $g_{n-1}(z)$ is a polynomial of degree $n-1$. Since, clearly, $\left|g_{n-1}(x)\right| \leqq 1$ for $-1 \leqq x \leqq 1$, Theorem A gives

$$
\left|p_{n}^{\prime}(x)\right| \leqq|x|\left|g_{n-1}^{\prime}(x)\right|+\left|g_{n-1}(x)\right| \leqq(n-1)^{2}+1
$$

for $-1 \leqq x \leqq 1$. Thus we have

THEOREM 5. If $p_{n}(z)$ is a polynomial of degree $n$ such that $\left|p_{n}(x)\right| \leqq|x|$ for $-1 \leqq x \leqq 1$ then

$$
\max _{-1 \leqq x \leqq 1}\left|p_{n}^{\prime}(x)\right| \leqq(n-1)^{2}+1
$$


The example $p_{n}(z)=z T_{n-1}(z)$, where $T_{n-1}(z)$ is the Chebyshev polynomial of the first kind of degree $n-1$, shows that (9) is sharp.

We also prove

THEOREM 6. If $p_{n}(x)$ is a polynomial of degree $\leqq n$ such that $\left|p_{n}(x)\right| \leqq|x|$ for $-1 \leqq x \leqq 1$ then for a fixed $x_{0}$ in $(-1,1)$ we have

$$
\left|p_{n}^{\prime}\left(x_{0}\right)\right| \leqq\left\{(n-1)^{2} x_{0}^{2}\left(1-x_{0}^{2}\right)^{-1}+1\right\}^{1 / 2} .
$$

The example $p_{n}(z)=e^{i \gamma} z T_{n-1}(z)$ shows that in (10) equality is attained at those points of the interval $-1<x<1$ where $\left(1-x^{2}\right)^{1 / 2} \tan \left\{(n-1) \cos ^{-1} x\right\}=(n-1) x$.

From a geometric point of view, those members of the class $P_{|x|}$ which are typically real [10] in $|z|<1$ constitute an interesting subclass. If we restrict ourselves to this subclass we can replace (9) by a considerably stronger inequality.

THEOREM 7. Let $p_{n}(z)$ be a polynomial of degree $n$ such that $\left|p_{n}(x)\right| \leqq|x|$ for $-1 \leqq x \leqq 1$. If $p_{n}(z)$ is typically real in $|z|<1$ then

$$
\left|p_{n}^{\prime}(x)\right| \leqq(n+1) / 2
$$

for $-1 \leqq x \leqq 1$.

A function $g(z)$ analytic in $|z|<1$ is typically real in $|z|<1$ if and only if [10, p. 112] $g(z)$ is real for real $z$ and $\operatorname{Re}\left\{\left(\left(1-z^{2}\right) / z\right) g(z)\right\} \neq 0$ in $|z|<1$. Hence if $n$ is odd the polynomial

$$
p_{n}(z)=2\left(z+z^{3}+\cdots+z^{n}\right) /(n+1)
$$

is typically real in $|z|<1$. It also belongs to $P_{|x|}$, and since $\left|p_{n}^{\prime}(1)\right|=(n+1) / 2$ the bound in (11) cannot in general be improved.

Lemmas. We shall now collect some results which we shall use in the proofs of the above theorems.

If $p_{n}(x)$ is a polynomial of degree $n$ then $p_{n}(\cos \theta)$ is a trigonometric polynomial of degree $n$. Since $d \theta=-\left(1-x^{2}\right)^{-1 / 2} d x$, inequality (2) states that $\left|(d / d \theta) p_{n}(\cos \theta)\right|$ $\leqq n$. Hence Theorem $\mathrm{B}$ is a consequence of the following result known as Bernstein's theorem for trigonometric polynomials.

THEOREM D. If $t(\theta)$ is a trigonometric polynomial of degree $n$ and $|t(\theta)| \leqq 1$, then $\left|t^{\prime}(\theta)\right| \leqq n$.

It has been remarked by Boas [4, p. 169] that Markov's theorem (Theorem A) would also follow from Bernstein's theorem for trigonometric polynomials if it could be shown that $\left|p_{n}^{\prime}(x)\right|$ attains its maximum at \pm 1 if $p_{n}(x)$ is extremal.

We observe that the following result which is a refined version of Bernstein's theorem for trigonometric polynomials and which was independently proved by Szegö [12, p. 69], Boas [3, p. 287], van der Corput and Schaake [6, p. 321] is more appropriate for the study of polynomials on the unit interval and gives Markov's theorem as an immediate corollary. 
LEMMA 1. Let $t(\theta)=\sum_{k=-n}^{n} a_{k} e^{i k \theta}$ be a real trigonometric polynomial of degree $n$. If $|t(\theta)| \leqq 1$ then

$$
n^{2}\{t(\theta)\}^{2}+\left\{t^{\prime}(\theta)\right\}^{2} \leqq n^{2} .
$$

This result plays a central role in our paper. First of all we use it to prove:

LeMma 2. If $p_{n-1}(x)$ is a real valued polynomial of degree $n-1$ such that $\left(1-x^{2}\right)^{1 / 2}\left|p_{n-1}(x)\right| \leqq 1$ for $-1<x<1$ then

$$
\left|p_{n-1}(x)\right| \leqq n \text { for }-1 \leqq x \leqq 1 .
$$

Our hypothesis implies that $(\sin \theta) p_{n-1}(\cos \theta)$ is a real trigonometric polynomial of degree $n$ whose absolute value does not exceed 1. Hence according to Lemma 1

$$
n^{2} \sin ^{2} \theta\left\{p_{n-1}(\cos \theta)\right\}^{2}+\left\{(\cos \theta) p_{n-1}(\cos \theta)+(\sin \theta)(d / d \theta) p_{n-1}(\cos \theta)\right\}^{2} \leqq n^{2}
$$

for real $\theta$. At a point where $\left|p_{n-1}(\cos \theta)\right|$ attains its maximum value, $(d / d \theta) p_{n-1}(\cos \theta)$ must vanish. Consequently, at such a point $\theta_{0}$,

or

$$
n^{2}\left(\sin ^{2} \theta_{0}\right)\left\{p_{n-1}\left(\cos \theta_{0}\right)\right\}^{2}+\left(\cos ^{2} \theta_{0}\right)\left\{p_{n-1}\left(\cos \theta_{0}\right)\right\}^{2} \leqq n^{2}
$$

$$
\left(n^{2}-1\right)\left(\sin ^{2} \theta_{0}\right)\left\{p_{n-1}\left(\cos \theta_{0}\right)\right\}^{2}+\left\{p_{n-1}\left(\cos \theta_{0}\right)\right\}^{2} \leqq n^{2} .
$$

Therefore $\left|p_{n-1}\left(\cos \theta_{0}\right)\right| \leqq n$ which gives the desired result.

For the sake of completeness we include a proof of Lemma 1. In this way we will also be giving a complete and independent proof of Markov's theorem (since it follows from Theorem B in conjunction with Lemma 2). It may be noted that our proof of Lemma 1 depends only on the maximum modulus principle and the Gauss-Lucas theorem [8, p. 84].

Proof of Lemma 1. Let $p(z)$ be a polynomial of degree $m$ such that $|p(z)| \leqq M$ for $|z| \leqq 1$. Then for $|\lambda|>1$ the polynomial $P(z)=p(z)-\lambda M$ does not vanish in $|z| \leqq 1$. Let

$$
Q(z)=z^{m} \overline{P(1 / \bar{z})}=z^{m} \overline{p(1 / \bar{z})}-\bar{\lambda} M z^{m}=q(z)-\bar{\lambda} M z^{m} .
$$

Since the function $Q(z) / P(z)$ is holomorphic in $|z| \leqq 1$ and $|Q(z)|=|P(z)|$ for $|z|=1$ it follows from the maximum modulus principle that $|Q(z) / P(z)| \leqq 1$ for $|z| \leqq 1$. Replacing $z$ by $1 / \bar{z}$ we conclude that $|P(z)| \leqq|Q(z)|$ for $|z| \geqq 1$. Thus for $|\mu|>1$ all the zeros of the polynomial $P(z)-\mu Q(z)$ lie in $|z|<1$ and so do the zeros of $P^{\prime}(z)-\mu Q^{\prime}(z)$ by Gauss-Lucas theorem. Consequently,

$$
\left|p^{\prime}(z)\right|=\left|P^{\prime}(z)\right| \leqq\left|Q^{\prime}(z)\right|=\left|q^{\prime}(z)-\lambda M m z^{m-1}\right| \text { for }|z| \geqq 1 .
$$

According to our hypothesis $|p(z)| \leqq M$ for $|z| \leqq 1$. Since

we have

$$
p(z) \equiv z^{m} \overline{q(1 / \bar{z})}
$$

$$
\left|z^{m} \overline{q(1 / \bar{z})}\right| \leqq M \text { for }|z| \leqq 1,
$$


i.e., $|q(z)| \leqq M|z|^{m}$ for $|z| \geqq 1$. Hence for $|\Lambda|>1$ all the zeros of the polynomial $q(z)-\Lambda M z^{m}$ lie in $|z|<1$ and by the Gauss-Lucas theorem so do the zeros of $q^{\prime}(z)-\Lambda M m z^{m-1}$. This implies that $\left|q^{\prime}(z)\right| \leqq M m|z|^{m-1}$ for $|z| \geqq 1$. Given a point $z$ in the circular domain $|z| \geqq 1$, this inequality permits us to choose $\arg \lambda$ in (14) such that $\left|q^{\prime}(z)-\lambda M m z^{m-1}\right|=|\lambda| M m|z|^{m-1}-\left|q^{\prime}(z)\right|$. We readily obtain

$$
\left|p^{\prime}(z)\right|+\left|q^{\prime}(z)\right| \leqq M m|z|^{m-1} \text { for }|z| \geqq 1
$$

In particular, we have

$$
\left|(d / d \theta) p\left(e^{i \theta}\right)\right|+\left|-i m p\left(e^{i \theta}\right)+(d / d \theta) p\left(e^{i \theta}\right)\right| \leqq M m .
$$

If $t(\theta)=\sum_{k=-n}^{n} a_{k} e^{i k \theta}$ is a trigonometric polynomial of degree $n$ and $|t(\theta)| \leqq 1$ then $e^{i n \theta} t(\theta)=p\left(e^{i \theta}\right)$ where $p(z)$ is a polynomial of degree $2 n$ such that $|p(z)| \leqq 1$ for $|z| \leqq 1$. From (16) we get $\left|\operatorname{int}(\theta)+t^{\prime}(\theta)\right|+\left|-\operatorname{int}(\theta)+t^{\prime}(\theta)\right| \leqq 2 n$. Hence $n^{2}\{t(\theta)\}^{2}$ $+\left\{t^{\prime}(\theta)\right\}^{2} \leqq n^{2}$ if the trigonometric polynomial is real.

For the proof of Theorem 7 we shall need the following result due to de Bruijn [5, Theorem 4].

LEMma 3. Let $C$ be a circular domain in the z-plane, and $S$ an arbitrary point set in the w-plane. If the polynomial $P(z)$ of degree $n$ satisfies $P(z)=w \in S$ for any $z \in C$, then we have, for any $z \in C$ and any $\xi \in C$,

$$
(\xi / n) P^{\prime}(z)+P(z)-z P^{\prime}(z) / n \in S .
$$

\section{Proofs of the theorems.}

Proof of Theorem 1. As remarked earlier there is no loss of generality in assuming $p_{n}(x)$ to be real-valued. Since $p_{n}(x)$ vanishes at the points $-1,+1$ we have $p_{n}(x)$ $=\left(1-x^{2}\right) q_{n-2}(x)$ where $q_{n-2}(x)$ is a polynomial of degree $n-2$. We set $\left(1-x^{2}\right)^{1 / 2} q_{n-2}(x)=f(x)$ and write $p_{n}(x)$ as the product of $\left(1-x^{2}\right)^{1 / 2}$ and $f(x)$. Thus

$$
\begin{aligned}
\left|p_{n}^{\prime}(x)\right| & =\left|-x\left(1-x^{2}\right)^{-1 / 2} f(x)+\left(1-x^{2}\right)^{1 / 2} f^{\prime}(x)\right| \\
& \leqq|x|\left|\left(1-x^{2}\right)^{-1 / 2} f(x)\right|+\left|\left(1-x^{2}\right)^{1 / 2} f^{\prime}(x)\right| .
\end{aligned}
$$

We observe that $f(\cos \theta)$ is a trigonometric polynomial of degree $n-1$ whose absolute value does not exceed 1 . Hence according to Theorem D $|(d / d \theta) f(\cos \theta)| \leqq n-1$ for real $\theta$. Since

$$
(-\sin \theta) \frac{d}{d(\cos \theta)} f(\cos \theta)=\frac{d}{d \theta} f(\cos \theta)
$$

we get

$$
\left|\left(1-x^{2}\right)^{1 / 2} f^{\prime}(x)\right| \leqq n-1 \text { for }-1 \leqq x \leqq 1 .
$$

Now let us note that $\left(1-x^{2}\right)^{-1 / 2} f(x)$ is a polynomial of degree $n-2$ such that $\left|\left(1-x^{2}\right)^{1 / 2} \cdot\left(1-x^{2}\right)^{-1 / 2} f(x)\right|=|f(x)| \leqq 1$ for $-1 \leqq x \leqq 1$. Hence by Lemma 2

$$
\left|\left(1-x^{2}\right)^{-1 / 2} f(x)\right| \leqq n-1 \text { for }-1 \leqq x \leqq 1 .
$$


Using (18) and (19) in (17) we get the desired estimate for $\max _{-1 \leqq x \leqq 1}\left|p_{n}^{\prime}(x)\right|$.

REMARK. Our proof of Theorem 1 makes particular use of the fact that the polynomial $p_{n}(z)$ under consideration vanishes at the points $-1,+1$. However, the bound for $\max _{-1 \leqq x \leqq 1}\left|p_{n}^{\prime}(x)\right|$ is not very much improved if we only add this requirement to the hypothesis in Markov's theorem. By considering the polynomial $p_{n}(x)=\cos n \cos ^{-1}(x \cos (\pi / 2 n))$ we see that $\max _{-1 \leqq x \leqq 1}\left|p_{n}^{\prime}(x)\right|$ can be as large as $n \cot (\pi / 2 n)$ if $p_{n}( \pm 1)=0$ and $\max _{-1 \leqq x \leqq 1}\left|p_{n}(x)\right|=1$. A theorem of Schur [11, pp. 284-285] says that $\max _{-1 \leqq x \leqq 1}\left|p_{n}^{\prime}(x)\right| \leqq n \cot (\pi / 2 n)$ for every polynomial of degree $\leqq n$ satisfying the inequality $\left|p_{n}(x)\right| \leqq 1$ for $-1 \leqq x \leqq 1$ and vanishing at the points $-1,+1$.

Proof of Theorem 2. Without loss of generality we may assume $p_{n}(x)$ to be realvalued. Again setting $f(x)=\left(1-x^{2}\right)^{-1 / 2} p_{n}(x)$ we see that $f(\cos \theta)$ is a real trigonometric polynomial of degree $n-1$ whose absolute value does not exceed 1 . Hence from Lemma 1

$$
(n-1)^{2} f^{2}(x)+\left(1-x^{2}\right)\left\{f^{\prime}(x)\right\}^{2} \leqq(n-1)^{2} \text { for }-1 \leqq x \leqq 1 .
$$

Using this inequality in (17) we conclude that for $-1<x<1$

$$
\begin{aligned}
\left|p_{n}^{\prime}(x)\right| & \leqq|x|\left(1-x^{2}\right)^{-1 / 2}|f(x)|+(n-1)\left\{1-|f(x)|^{2}\right\}^{1 / 2} \\
& \leqq \max _{0 \leqq y \leqq 1}\left\{|x|\left(1-x^{2}\right)^{-1 / 2} y+(n-1)\left(1-y^{2}\right)^{1 / 2}\right\} .
\end{aligned}
$$

For a given $x$ in $(-1,1)$ the maximum of the expression $|x|\left(1-x^{2}\right)^{-1 / 2} y$ $+(n-1)\left(1-y^{2}\right)^{1 / 2}$ is $\left\{x^{2}\left(1-x^{2}\right)^{-1}+(n-1)^{2}\right\}^{1 / 2}$ which is attained when

$$
y=|x|\left\{(n-1)^{2}\left(1-x^{2}\right)+x^{2}\right\}^{-1 / 2} .
$$

Proof of Theorem 3. We have

$$
\left|a_{2}\right|=\frac{1}{2}\left|p_{n}^{\prime \prime}(0)\right|=\frac{1}{2}\left|f^{\prime \prime}(0)-f(0)\right| \leqq \frac{1}{2}\left\{\left|f^{\prime \prime}(0)\right|+|f(0)|\right\}
$$

where $f(x)=\left(1-x^{2}\right)^{-1 / 2} p_{n}(x)$. Now if $F(\theta)=f(\cos \theta)$ then $\left|f^{\prime \prime}(0)\right|=\left|F^{\prime \prime}(\pi / 2)\right|$ and hence by Theorem $\mathrm{D}\left|f^{\prime \prime}(0)\right| \leqq(n-1)^{2}$. Since $|f(0)| \leqq 1$ we get the desired result.

Proof of Theorem 4. This result is proved in exactly the same way as Theorem C was proved by Duffin and Schaeffer [7, p. 240]. We need only to observe that $f(\cos z)=(\operatorname{cosec} z) p_{n}(\cos z)$ is an entire function of exponential type $n-1$.

If $p_{n}(z)=\left(1-z^{2}\right) U_{n-2}(z)$, then (8) becomes an equality at the points

$$
z=\left(\left(R+R^{-1}\right) / 2\right) \cos \phi_{k} \pm i\left(\left(R-R^{-1}\right) / 2\right) \sin \phi_{k}
$$

where $\phi_{k}=\left\{\left(2 k+(-1)^{k}\right) / 2(n-1)\right\} \pi, k=0,1,2, \ldots$

Proof of Theorem 6. It is enough to prove the theorem for polynomials which assume real values on the real axis. We have $p_{n}(x)=x g_{n-1}(x)$ where $g_{n-1}(x)$ is a polynomial of degree $n-1$ which assumes real values for real $x$ and $\left|g_{n-1}(x)\right| \leqq 1$ for $-1 \leqq x \leqq 1$. Thus $g_{n-1}(\cos \theta)$ is a real trigonometric polynomial of degree $n-1$ such that $\left|g_{n-1}(\cos \theta)\right| \leqq 1$. Hence from Lemma 1 we get

$$
(n-1)^{2}\left\{g_{n-1}(x)\right\}^{2}+\left(1-x^{2}\right)\left\{g_{n-1}^{\prime}(x)\right\}^{2} \leqq(n-1)^{2} \quad \text { for }-1 \leqq x \leqq 1 .
$$


We use this inequality in $\left|p_{n}^{\prime}(x)\right| \leqq\left|g_{n-1}(x)\right|+|x|\left|g_{n-1}^{\prime}(x)\right|$ to complete the proof of the theorem in precisely the same way as for Theorem 2 .

Proof of Theorem 7. According to hypothesis $p_{n}(z)$ assumes real values in $|z|<1$ if and only if $z$ is real. Hence $p_{n}^{\prime}(x) \neq 0$ for $-1<x<1$ and $p_{n}(x)$ is a monotonic function on the interval $-1 \leqq x \leqq 1$. Without loss of generality we may suppose $p_{n}(x)$ to be increasing on $[-1,1]$. Let $x_{0}$ be a given point of the open interval $(0,1)$. The polynomial $P(z)=p_{n}\left(x_{0} z\right)$ is typically real in $|z|<1 / x_{0}$ and hence in $|z| \leqq 1$. Also $|P(x)| \leqq x_{0}$ for $-1 \leqq x \leqq 1$. Since the only zero of $P(z)$ in $|z|<\left|x_{0}\right|^{-1}$ is a simple zero at the origin, $Q(z)=z^{n} P\left(z^{-1}\right)$ is a polynomial of degree $n-1$ having all its zeros in $|z| \leqq x_{0}$. Hence according to Walsh's generalization of Laguerre's theorem [13, Lemma 1, p. 13] $Q^{\prime}(1) / Q(1)=(n-1) /(1-w)$ where $|w| \leqq x_{0}$. Consequently $\left|Q^{\prime}(1)\right| \geqq\left((n-1) /\left(1+x_{0}\right)\right)|Q(1)|$, i.e.

$$
\left|n p_{n}\left(x_{0}\right)-x_{0} p_{n}^{\prime}\left(x_{0}\right)\right| \geqq \frac{n-1}{1+x_{0}}\left|p_{n}\left(x_{0}\right)\right|=\frac{n-1}{1+x_{0}} p_{n}\left(x_{0}\right) .
$$

But if $z_{1}, z_{2}, \ldots, z_{n-1}$ are the zeros of $z^{-1} p_{n}(z)$ then

$$
\frac{x_{0} p_{n}^{\prime}\left(x_{0}\right)}{p_{n}\left(x_{0}\right)}=\operatorname{Re}\left\{\frac{x_{0} p_{n}^{\prime}\left(x_{0}\right)}{p_{n}\left(x_{0}\right)}\right\}=1+\sum_{j=1}^{n-1} \operatorname{Re}\left(\frac{x_{0}}{x_{0}-z_{j}}\right)
$$

where $\operatorname{Re}\left(x_{0} /\left(x_{0}-z_{j}\right)\right) \leqq \frac{1}{2}, \quad 1 \leqq j \leqq n-1$, since $\left|z_{j}\right| \geqq 1$. Thus $n p_{n}\left(x_{0}\right)-x_{0} p_{n}^{\prime}\left(x_{0}\right)$ $\geqq \frac{1}{2}(n-1) p_{n}\left(x_{0}\right) \geqq 0$, and $(22)$ can be written as

$$
n p_{n}\left(x_{0}\right)-x_{0} p_{n}^{\prime}\left(x_{0}\right) \geqq \frac{n-1}{1+x_{0}} p_{n}\left(x_{0}\right) .
$$

This implies that the point $p_{n}\left(x_{0}\right)-n^{-1} x_{0} p_{n}^{\prime}\left(x_{0}\right)$ lies on the interval

$$
\left[\left(1-n^{-1}\right)\left(1+x_{0}\right)^{-1} p_{n}\left(x_{0}\right), p_{n}\left(x_{0}\right)\right] \text {. }
$$

Now we note that the image $S$ of the circular domain $|z| \leqq x_{0}$ under the mapping $w=p_{n}(z)$ lies in the plane cut along the positive real axis from $p_{n}\left(x_{0}\right)$ to infinity. According to Lemma 3 the disk

$$
\left|w-\left\{p_{n}\left(x_{0}\right)-n^{-1} x_{0} p_{n}^{\prime}\left(x_{0}\right)\right\}\right| \leqq n^{-1} x_{0} p_{n}^{\prime}\left(x_{0}\right)
$$

lies in $S$. This is possible only if

$$
n^{-1} x_{0} p_{n}^{\prime}\left(x_{0}\right) \leqq p_{n}\left(x_{0}\right)\left\{1-\left(1-n^{-1}\right)\left(1+x_{0}\right)^{-1}\right\} .
$$

Since $p_{n}\left(x_{0}\right) \leqq x_{0}$ we get

$$
p_{n}^{\prime}\left(x_{0}\right) \leqq n-(n-1)\left(1+x_{0}\right)^{-1} .
$$

By continuity $p_{n}^{\prime}(0) \leqq 1$ and $p_{n}^{\prime}(1) \leqq(n+1) / 2$. Hence

$$
\max _{0 \leqq x \leqq 1} p_{n}^{\prime}(x) \leqq(n+1) / 2 .
$$


Applying this result to $-p_{n}(-x)$ we get

$$
\max _{-1 \leqq x \leqq 0} p_{n}^{\prime}(x) \leqq(n+1) / 2 .
$$

The desired result follows from (24) and (25).

Inequality (23) gives an estimate for $\left|p_{n}^{\prime}\left(x_{0}\right)\right|$ at a fixed point $x_{0}$ in $[-1,1]$ but the bound does not appear to be sharp except at $-1,0,+1$.

I wish to thank Professor P. Erdös for telling me about the problem of Turán.

\section{REFERENCES}

1. L. V. Ahlfors, Complex analysis: An introduction to the theory of analytic functions of one complex variable, 2nd ed., McGraw-Hill, New York, 1966. MR 32 \#5844.

2. S. Bernstein, Sur l'ordre de la meilleure approximation des fonctions continues par des polynômes de degré donné, Mém. Acad. Roy. Belg. (2) 4 (1912), 1-103.

3. R. P. Boas, Some theorems on Fourier transforms and conjugate trigonometric integrals, Trans. Amer. Math. Soc. 40 (1936), 287-308.

4. - Inequalities for the derivatives of polynomials, Math. Mag. 42 (1969), 165-174.

5. N. G. de Bruijn, Inequalities concerning polynomials in the complex domain, Nederl. Akad. Wetensch. Proc. Ser. A. 50 (1947), 1265-1272=Indag. Math. 9 (1947), 591-598.

6. J. G. van der Corput and G. Schaake, Ungleichungen für Polynome und trigonometrische Polynome, Compositio Math. 2 (1935), 321-361.

7. R. Duffin and A. C. Schaeffer, Some properties of functions of exponential type, Bull. Amer. Math. Soc. 44 (1938), 236-240.

8. E. Hille, Analytic function theory. Vol. 1, Introduction to Higher Math., Ginn, Boston, Mass., 1959. MR 21 \#6415.

9. A. Markov, On a problem of D. I. Mendeleev, Zap. Imp. Akad. Nauk 62 (1889), 1-24. (Russian)

10. W. Rogosinski, Über positive harmonische Entwicklungen und typisch-reelle Potenzreihen, Math. Z. 35 (1932), 93-121.

11. I. Schur, Über das Maximum des absoluten Betrages eines Polynoms in einem gegebenen Intervall, Math. Z. 4 (1919), 271-287.

12. G. Szegö, Über einen Satz des Herrn Serge Bernstein, Schr. Königsberg. Gel. Ges. 5 (1928), 59-70.

13. J. L. Walsh, The location of critical points of analytic and harmonic functions, Amer. Math. Soc. Colloq. Publ., vol. 34, Amer. Math. Soc., Providence, R. I., 1950. MR 12, 249.

Université de Montréal, Montréal, Quebec, Canada 